\title{
Elongation and orientation of macropores by shear stress in silica-polymer composite I. Effects of shear time and shear rate
}

\author{
Takeshi HIGUCHI, ${ }^{\dagger}$ Haruna OIZUMI, Tatsuya SASAKI and Tadahiro AITA \\ Department of Chemistry and Chemical Engineering, Yamagata University, 4-3-16 Jonan, Yonezawa, Yamagata $992-8510$
}

\begin{abstract}
A silica-polymer composite with oriented macropores was fabricated from tetraethoxysilane (TEOS) incorporated with poly(ethylene glycol) (PEG) via a sol-gel method. The solution mixture containing PEG and TEOS after hydrolysis was subjected to shear stress so that the macroporous structure generated by spinodal decomposition during the process of polycondensation to a silica-PEG-rich phase and a solvent-rich phase was elongated and oriented toward the shear flow direction. It was found that shear flow is effective for macropore elongation after the solution mixture becomes turbid. The optimum PEG content for macropore elongation was also revealed. The elongation ratio of the macropores was dependent to a certain extent on the shear rate.
\end{abstract}

(C2009 The Ceramic Society of Japan. All rights reserved.

Key-words : Sol-gel method, Spinodal decomposition, Porous silica, Shear stress, Elongation, Orientation

[Received February 9, 2009; Accepted July 16, 2009]

\section{Introduction}

Silica-polymer macroporous composites (MPCs) have recently attracted much interest in industrial and analytical fields. One of the most successful applications of MPCs is as a monolithic column rod for HPLC. ${ }^{1)-5)}$ A comprehensive study of MPC fabrication through a sol-gel process from alkoxysilane was performed by Nakanishi and colleagues. ${ }^{6-11)}$ MPCs have an interconnected macroporous structure, whose pore diameter is on the submicron to micron scale, generated by a bicontinuous twophase separation caused by spinodal decomposition during the polycondensation and gelation stages of silica.

The macroporous structure mentioned above is inherently isotropic because MPCs are amorphous and the mechanism of spinodal decomposition provides no macroscopic information on the direction of diffusion, which enhances concentration fluctuation. Considering the application of MPCs to a filter or a column, a straight and well-aligned macroporous structure is preferable because of the lower pressure drop of an inner fluid than of an isotropic random macropore. The formation of such a structure via spinodal decomposition might be a novel and promising method for producing a functional material with an ordered structure. Loading shear stress on a bicontinuous phase structure is a possible means of obtaining straight macropores by elongating the phase structure through spinodal decomposition. How shear stress affects phase structure evolution and rheological behavior has been studied mainly in work on polymer blend ${ }^{12-24)}$ and semidilute polymer solution. ${ }^{25)}$ In this study, the authors attempted to fabricate MPCs with an elongated and oriented macroporous structure by loading shear stress on the solution mixture during the polycondensation and gelation stages, where spinodal decomposition proceeds concurrently.

Corresponding author: T. Higuchi; E-mail: higuchi@yz.yamagatau.ac.jp

\section{Experimental procedure}

\subsection{Chemicals}

Tetraethoxysilane (TEOS), which was used as the silica source, was purchased from Shin-Etsu Chemical Co., Ltd. Poly(ethylene glycol) (PEG, MW = 20000) and $1 \mathrm{~mol} \mathrm{dm}^{-3}$ nitric acid were from Wako Pure Chemical Industries, Ltd. All the chemicals were used without further purification.

\subsection{MPC preparation}

A typical procedure for MPC preparation is shown in Fig. 1.

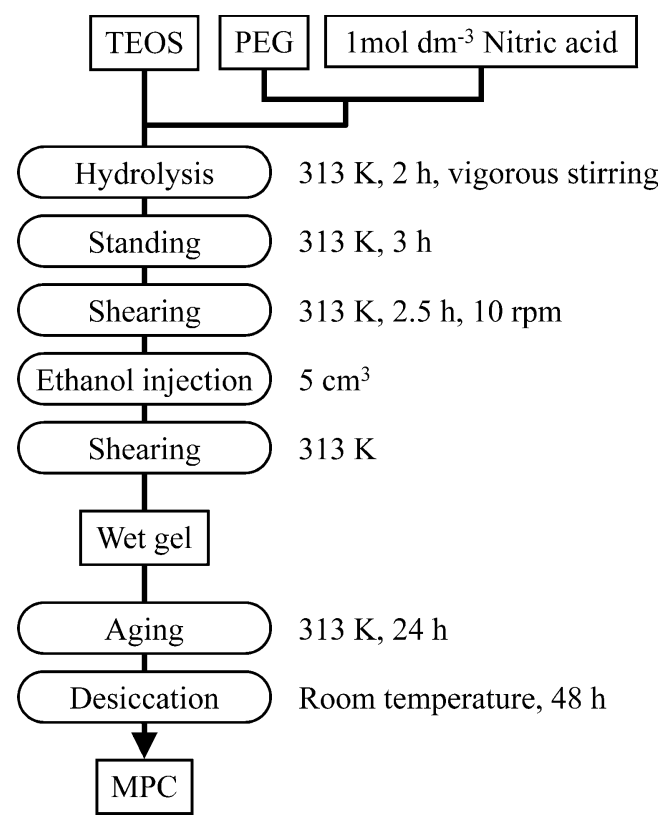

Fig. 1. Procedure for MPC preparation. 
Briefly, $6.5 \mathrm{~g}$ of TEOS was mixed with $7.7 \mathrm{~cm}^{3}$ of $1 \mathrm{~mol} \mathrm{dm}$ nitric acid containing $0.8 \mathrm{~g}$ of PEG, unless otherwise noted. The solution mixture was stirred vigorously and hydrolyzed for $2 \mathrm{~h}$ to a homogeneous transparent solution. The solution was sealed and left to stand for $3 \mathrm{~h}$ before subjecting to shear stress.

Shear stress was loaded on the solution as shown in Fig. 2. The solution was poured into a petri dish with an $80 \mathrm{~mm}$ inner diameter placed on a hot plate. A rotation disk was placed on the solution so as to form contact with its surface. First, shear stress was loaded at a rotation rate of $10 \mathrm{rpm}$ for $2.5 \mathrm{~h}$, turning the solution turbid. Then, $5 \mathrm{~cm}^{3}$ of ethanol was gently injected on the surface of the solution after slightly lifting the rotation disk from the surface. Rotation was started again after the disk was laid onto the surface; it was continued until the fluidity of the solution disappeared. The wet gel obtained was aged for $24 \mathrm{~h}$ in a sealed petri dish. All the processes mentioned above were performed at $313 \mathrm{~K}$. Finally, the gel was desiccated for $48 \mathrm{~h}$ at ambient temperature, and an MPC was obtained.

\section{Results and discussion}

\subsection{Effect of ethanol injection on gel formation}

Figure 3 shows the three types of wet gel fabricated under different injection conditions. Without any liquid injections, many cracks were generated all over each of the resulting wet gels (Fig. $3(\mathrm{a})$ ). The injection of $5 \mathrm{~cm}^{3}$ of ethanol immediately after the solution turned turbid successfully formed a homogenous monolithic wet gel (Fig. 3(b)). By injecting $5 \mathrm{~cm}^{3}$ of $1 \mathrm{~mol} \mathrm{dm}^{-3}$ nitric acid instead of ethanol, the solution gelled immediately and an agglomerated gel was obtained (Fig. 3(c)). As polycondensation progressed, the fluidity of the solution decreased gradually, and after gelation no longer followed the rotation of the disk. Had there been no liquid layer between the gelling solution and the

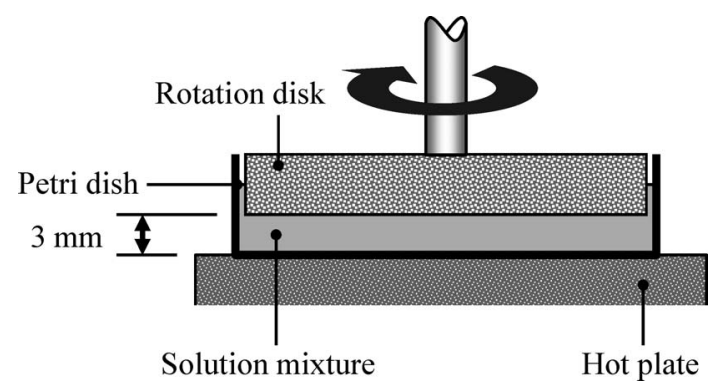

Fig. 2. Schematic of shear stress loading. After $2.5 \mathrm{~h}, 5 \mathrm{~cm}^{3}$ of ethanol was injected between the rotation disk and the solution mixture. Then the thickness of the shear layer (distance between the rotation disk and the bottom of the petri dish) became $3.5 \mathrm{~mm}$ rotation disk, the gel would have been broken by shear stress. The ethanol layer prevented the collapse of the wet gel by absorbing shear stress, as shown in Fig. 4. The rapid gelation when nitric acid was injected could be attributed to the catalytic behavior of nitric acid.

The injected ethanol layer plays as "lubricant", a buffer of excess shear stress. Although immiscible fluid such as fatty oil or silicone oil might be better alternative as lubricant, miscible liquids were used for ease of washing after gelation. Ethanol and nitric acid are already contained in the solution after hydrolysis and therefore, it is expected that influence of diffusion between the gelling solution and the injected liquid is relatively small. Ethanol is suitable as "lubricant" because it is inert to hydrolysis and polycondensation compared to nitric acid and water.

\subsection{Effects of starting composition on macropo- rouos structure and its elongation}

The macroporous structure of MPCs strongly depends on their starting composition (Table 1). Generally, if the solution is not subjected to shear stress, a higher fraction of silica results in isolated macropores. Otherwise, a bicontinuous network-like structure or a particle stacking structure can be observed. Figure 5 shows SEM images of MPCs without loading shear stress. The macroporous structure was "interconnected" in (a) and "isolated" in (b), respectively. Figure 6 shows macroscopic and SEM images of MPCs and the wet gel with loading shear stress. The starting compositions of (c) and (d), which resulted in an isolated macroporous structure when no shear stress was loaded (no SEM images are shown), hardly elongated macropores in spite of loading shear stress. A broken wet gel was obtained with the starting

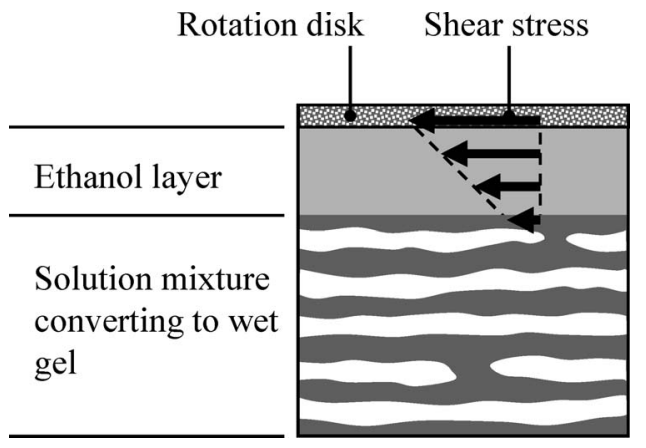

Fig. 4. Schematic of ethanol layer preventing gel collapse. The ethanol layer absorbs shear stress after the solution mixture loses fluidity by gelation.
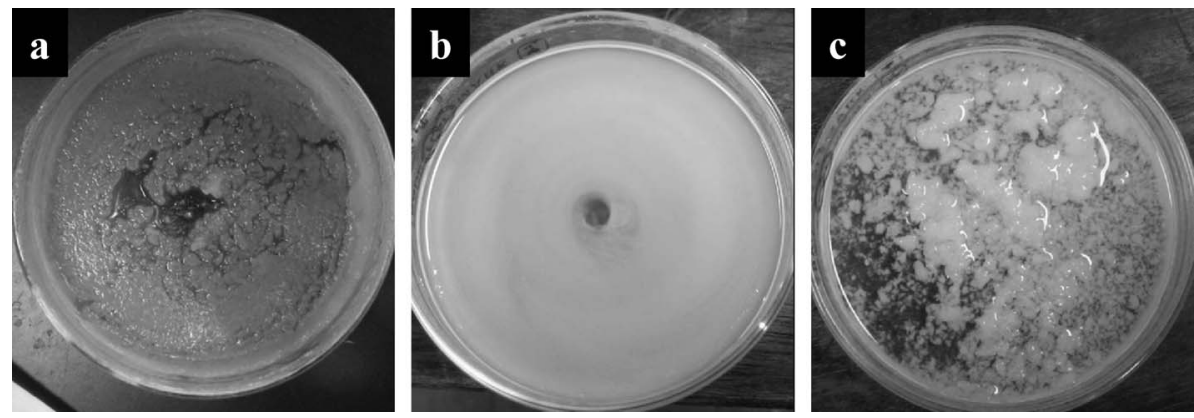

Fig. 3. Macroscopic images of wet gels after shear stress loading (a) without liquid injection, and with injection with (b) $5 \mathrm{~cm}^{3}$ of ethanol and (c) $5 \mathrm{~cm}^{3}$ of $1 \mathrm{~mol} \mathrm{dm}^{-3}$ nitric acid. 
Table 1. Starting Compositions of MPCs

\begin{tabular}{rrrcccc}
\hline & \multicolumn{2}{c}{ TEOS } & \multicolumn{2}{c}{ PEG } & \multicolumn{2}{c}{ Nitric acid } \\
& \multicolumn{1}{c}{$(\mathrm{g})$} & $(\mathrm{w} / \mathrm{w})$ & $(\mathrm{g})$ & $(\mathrm{w} / \mathrm{w})$ & $(\mathrm{g})$ & $(\mathrm{w} / \mathrm{w})$ \\
\hline (a) & 6.5 & 0.43 & 0.8 & 0.05 & 7.7 & 0.52 \\
(b) & 6.5 & 0.44 & 0.6 & 0.04 & 7.7 & 0.52 \\
(c) & 11.5 & 0.57 & 0.8 & 0.04 & 7.7 & 0.39 \\
(d) & 6.5 & 0.64 & 0.8 & 0.08 & 2.8 & 0.28 \\
\hline
\end{tabular}

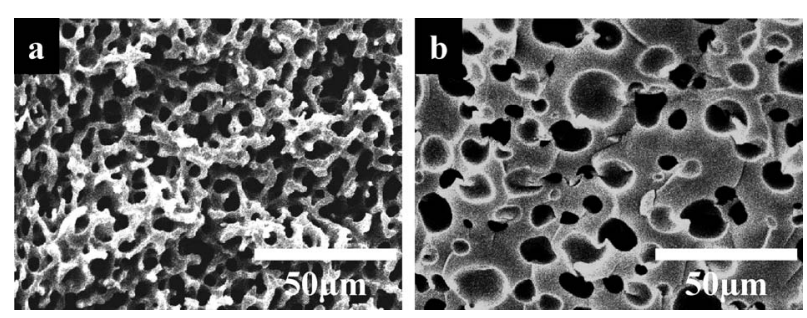

Fig. 5. SEM image of MPCs without loading shear stress. Starting compositions are shown in Table 1.
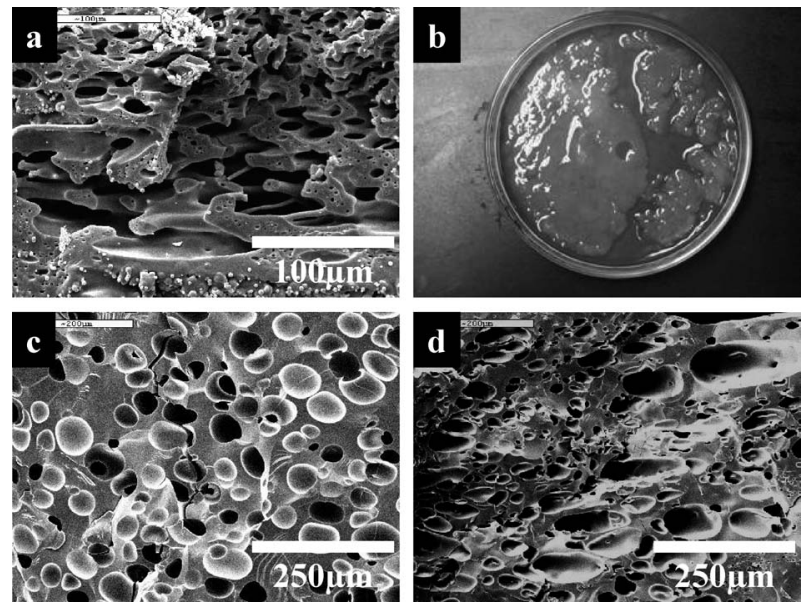

Fig. 6. Macroscopic and SEM images of MPCs (a), (c), (d) and wet gel (b) with loading shear stress. Starting compositions are shown in Table 1.

composition of (b).

Comparing MPCs without loading shear stress with those with loading shear stress (Fig. 5(a)), their macropores not only were elongated but also enlarged to several tens of micrometers in the short axis (Fig. 6(a)). This suggests that macropore merging and rearrangement occurred when MPCs were subjected to shear stress.

\subsection{Macropore elongation and orientation by shear stress}

The SEM image of MPCs was converted to a black-and-white binarized image before superimposing the grid pattern. All grid lines had the same span and the same length so that each section and the whole grid area were square. The numbers of intersections of the vertical and horizontal grid lines with the edge of the black-and-white pattern were determined, as depicted in Fig. 7. To evaluate the degree of macropore elongation, the elongation ratio $E$ was introduced, which is defined as

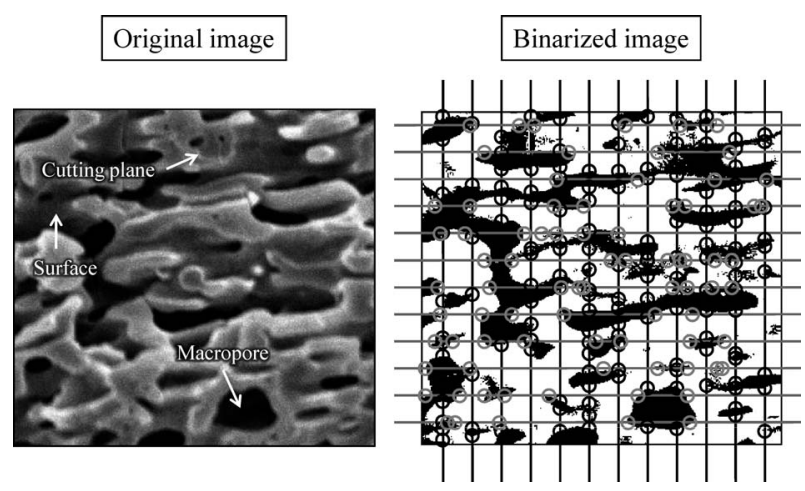

Fig. 7. Schematic of determining elongation ratio. Black and gray circles are intersections of the vertical and horizontal grid lines with the edge of the binarized pattern, respectively.

$$
E=\frac{N_{\mathrm{V}}}{N_{\mathrm{H}}}
$$

where $N_{\mathrm{V}}$ and $N_{\mathrm{H}}$ are the numbers of intersections of the vertical and horizontal grid lines with the edge of the black-and-white pattern, respectively. This ratio coincides a ratio of average code lengths of black and white areas which are defined as segment lengths along the horizontal and vertical grid lines, respectively because $N_{\mathrm{V}}$ and $N_{\mathrm{H}}$ are inversely proportional to the each code length. As shown in Fig. 7, SEM image of MPC is composed of cutting plane and surface of MPC and macropore. To evaluate $E$ properly, the cutting plane should be assigned to white area and the surface and macropore should be black area by the binarization. Brightness difference between cutting plane and surface is so small and vague that threshold of the binarization might contain some minor errors.

SEM images and $E$ of MPCs under various shearing conditions are shown in Figs. 8 and 9, respectively. The starting composition is listed in Table 1 (a). The shearing conditions are shown in Table 2. Shear rate depends on not only rotation rate but also the radial position of MPCs. In this study, apparent shear rate, $\dot{\gamma}$, is defined as

$$
\dot{\gamma}=\frac{2 \pi n r}{d}
$$

where $d$ is the thickness of the shear layer (corresponding to the sum of the thicknesses of the solution and liquid layer), $n$ is the rotation rate, and $r$ is the distance from the center of rotation.

The effect of the shear rate $\dot{\gamma}$ was studied by (1) observing different $r$ positions of the same MPCs and (2) altering the rotation rate $n$ after ethanol injection. A larger $r$ position obviously resulted in a greater $E$ (Figs. 8(a) and (f)). This means that a larger $\dot{\gamma}$ elongates macropores more effectively. Large difference of viscosity between two phases such as spinodal decomposition of polymer blend having a large difference in molecular weights or semidilute polymer solution tends to form dispersed phase when large $\dot{\gamma}$ is imposed through spinodal decomposition so that shear stress stored in viscous phase relaxes efficiently. ${ }^{23), 25)}$ Spinodal decomposition between silica-PEG composite and aqueous solution in the present study can be regarded as a similar phenomenon. But relaxation in silica-PEG phase considered much faster than in most of liner polymer solution or blend because of rigidity of silica gel network and therefore, considerably higher $\dot{\gamma}$ is required for dispersed phase morphology. While a critical shear rate, above which stress term in time-dependent Ginzburg-Landau (TDGL) equation dominates diffusion term, is 

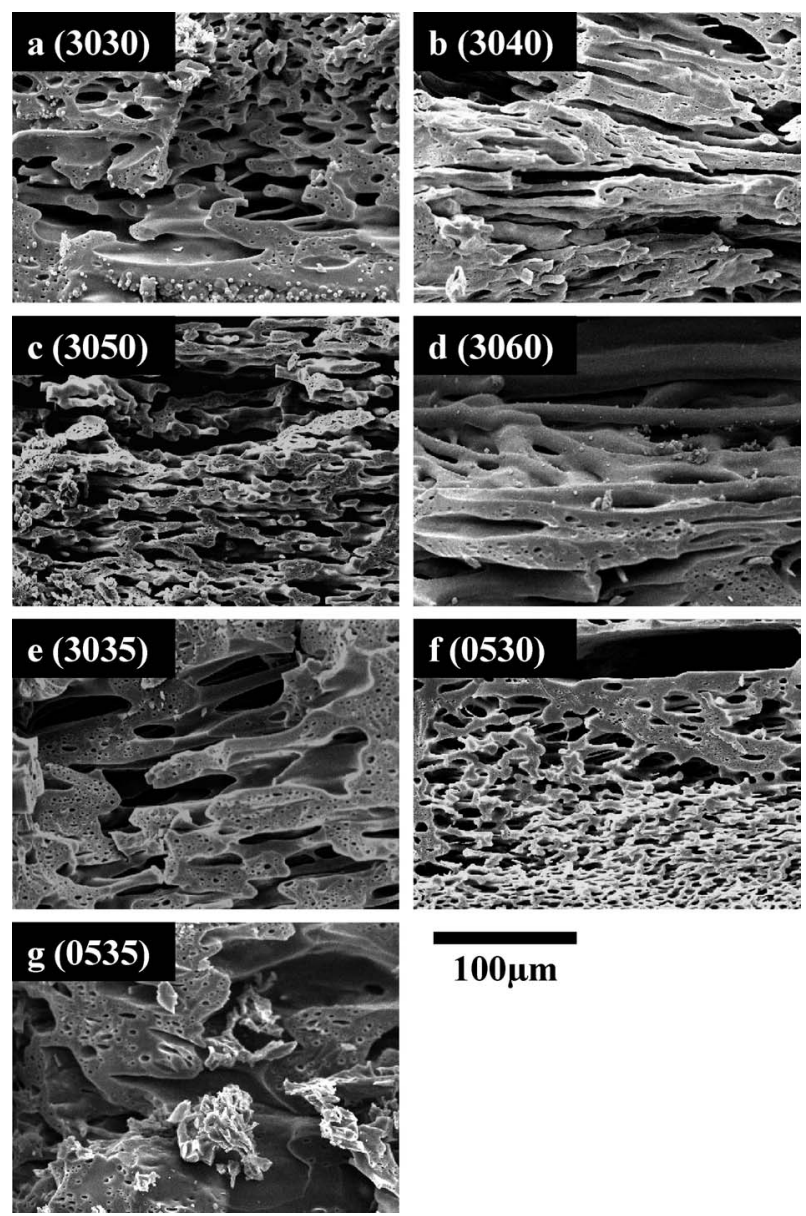

$100 \mu \mathrm{m}$

Fig. 8. SEM images of MPCs under various shearing conditions. Each number in brackets denotes the shearing condition listed in Table 2. Starting composition is listed in Table 1(a).

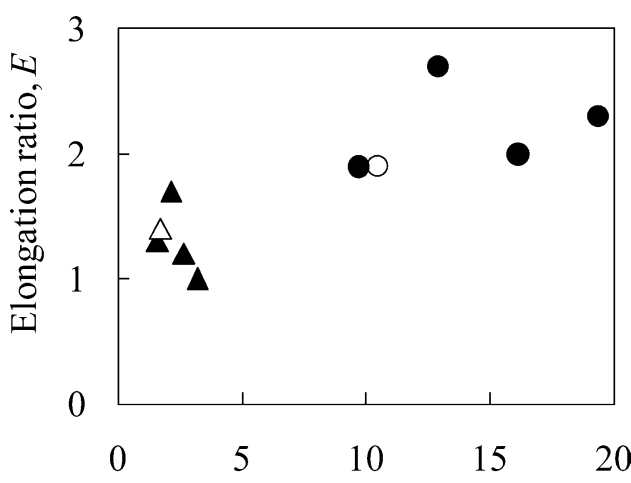

Shear strain, $\gamma / 10^{4}$

Fig. 9. Dependence of elongation ratio on shear strain. Circular and triangular markers denote distances of $30 \mathrm{~mm}$ and $5 \mathrm{~mm}$ from the center of rotation, respectively. Filled and open markers denote shearing disk rotation rates of $10 \mathrm{rpm}$ throughout polycondensation and gelation, and 10 rpm followed by $15 \mathrm{rpm}$ (listed in Table 2 (3035) and (0535)), respectively.

$2.5 \mathrm{~s}^{-1}$ for polystyrene - diethyl malonate semidilute system, ${ }^{25)}$ $\dot{\gamma}$ in the present study is $1.5-13 \mathrm{~s}^{-1}$. It is reasonable to suppose that $\dot{\gamma}$ is lower than or roughly the same as critical shear rate in this system. In such a situation, shearing promotes elongation of
Table 2. Shearing Conditions of MPCs

\begin{tabular}{|c|c|c|c|c|c|c|c|c|c|}
\hline \multirow[b]{2}{*}{ ID } & \multirow[b]{2}{*}{$\begin{array}{c}d^{\mathrm{a})} \\
(\mathrm{mm})\end{array}$} & \multirow[b]{2}{*}{$\begin{array}{c}r^{\mathrm{b})} \\
(\mathrm{mm})\end{array}$} & \multicolumn{3}{|c|}{ Before EtOH injection } & \multicolumn{3}{|c|}{ After EtOH injection } & \multirow[b]{2}{*}{$\begin{array}{c}\gamma^{\mathrm{f})} \\
\times 10^{-4}\end{array}$} \\
\hline & & & $\begin{array}{c}n^{\mathrm{c})} \\
(\mathrm{rpm})\end{array}$ & $\begin{array}{l}\dot{\gamma}^{\mathrm{d})} \\
\left(\mathrm{s}^{-1}\right)\end{array}$ & $t^{\mathrm{e})}(\mathrm{h})$ & $\begin{array}{c}n^{\mathrm{c})} \\
(\mathrm{rpm})\end{array}$ & $\begin{array}{c}\dot{\gamma}^{\mathrm{d})} \\
\left(\mathrm{s}^{-1}\right)\end{array}$ & $t^{\mathrm{e})}(\mathrm{h})$ & \\
\hline 3030 & 3.5 & 30 & 10 & 9.0 & 2.5 & 10 & 9.0 & 0.5 & 9.7 \\
\hline 3040 & & & & & & & & 1.5 & 13 \\
\hline 3050 & & & & & & & & 2.5 & 16 \\
\hline 3060 & & & & & & & & 3.5 & 19 \\
\hline 3035 & & & & & & 15 & 13 & 0.5 & 10 \\
\hline 0530 & & 5 & 10 & 1.5 & 2.5 & 10 & 1.5 & 0.5 & 1.6 \\
\hline 0540 & & & & & & & & 1.5 & 2.2 \\
\hline 0550 & & & & & & & & 2.5 & 2.7 \\
\hline 0560 & & & & & & & & 3.5 & 3.2 \\
\hline 0535 & & & & & & 15 & 2.2 & 0.5 & 1.8 \\
\hline
\end{tabular}

a) Thickness of shear layer corresponding to sum of thicknesses of solution mixture and liquid layer

b) Distance from center of rotation

c) Rotation rate of disk loading shear stress to solution mixture

d) Apparent shear rate defined in Eq. (2)

e) Shearing time

f) Total shear strain loaded to solution mixture defined as $\gamma=\dot{\gamma} t$.

the phase structure rather than suppress it. It would be safe to say that this is why $E$ was positively correlated with $\dot{\gamma}$.

Although the authors anticipated that prolonging $t$ and altering $n$ also positively affected on $E$ because greater quantity of $\gamma$ was expected to elongate the phase structure, the effects were not clear (Figs. 8(a) to (d) for $t$ and Figs. 8(a) and (e), (f) and (g) for $n)$. Although total shear strain $\gamma=\dot{\gamma} t$ was larger than $1 \times 10^{4}$ under every condition, $E$ did not exceed 3 . On the other hand, coarsened structure was observed in longer $t$ (Fig. 8(d)). These results can be interpreted as follows: The value of $E$ reached certain upper limit because further elongation was restricted by rigidity of silica gel network and interface tension between the two phases even long before structural freezing by gelation and after that, coarsening became predominant to minimize interface energy. Additionally, under the experimental conditions used in this study, $n$ could not be increased beyond $15 \mathrm{rpm}$ because wet gel breakage should be avoided. Therefore, the difference both in $\dot{\gamma}$ and $\gamma$ among the conditions with different $n$ could not be large enough to affect on the phase structure.

\section{Conclusion}

Macropores of silica-PEG composite were successfully elongated and oriented toward the shear direction. It was found that an ethanol layer injected when loading shear stress is necessary to avoid wet gel breakage. According to results of experiments with various shearing times and shear rates, the degree of macropore elongation was mainly dependent on the shear rate.

Acknowledgements The authors gratefully acknowledge financial support from the Saneyoshi Scholarship Foundation.

\section{References}

1) H. Minakuchi, K. Nakanishi, N. Soga, N. Ishizuka and N. Tanaka, Anal. Chem., 68, 3498-3501 (1996).

2) H. Minakuchi, K. Nakanishi, N. Soga, N. Ishizuka and N. 
Tanaka, J. Chromatography A, 797, 121-131 (1998).

3) H. Minakuchi, N. Ishizuka, K. Nakanishi, N. Soga and N. Tanaka, J. Chromatography A, 828, 83-90 (1998).

4) K. Nakanishi, H. Shikata, N. Ishizuka, N. Koheiya and N. Soga, J. High Resol. Chromatography, 23, 106-110 (2000).

5) N. Tanaka, H. Kobayashi, K. Nakanishi, H. Minakuchi and N. Ishizuka, Anal. Chem., 73, 420A-429A (2001).

6) K. Nakanishi, J. Porous Mater., 4, 67-112 (1997).

7) K. Nakanishi, H. Komura, R. Takahashi and N. Soga, Bull. Chem. Soc. Jpn., 67, 1327-1335 (1994).

8) K. Nakanishi and N. Soga, Bull. Chem. Soc. Jpn., 70, 587-592 (1997).

9) R. Takahashi, K. Nakanishi and N. Soga, J. Sol-Gel Sci. Technol., 17, 7-18 (2000).

10) K. Nakanishi, R. Takahashi, T. Nagakane, K. Kitayama, N. Koheiya, H. Shikata and N. Soga, J. Sol-Gel Sci. Tech., 17, 191-210 (2000).

11) Y. Sato, K. Nakanishi, K. Hirao, H. Jinnai, M. Shibayama, Y. B. Melnichenko and G. D. Wignall, Colloids Surf. A, 187, 117-122 (2001).

12) K. Matsuzaka, H. Jinnai, T. Koga and T. Hashimoto, Macromolecules, 30, 1146-1152 (1997).

13) H. Tanaka, Phys. Rev. E, 56, 4451-4462 (1997).
14) F. Qiu, H. Zhang and Y. Yang, J. Chem. Phys., 108, 95299536 (1998).

15) H. Gerard, J. S. Higgins and N. Clarke, Macromolecules, 32 , 5411-5422 (1999).

16) Z. L. Zhang, H. D. Zhang, Y. L. Yang, I. Vinckier and H. M. Laun, Macromolecules, 34, 1416-1429 (2001).

17) K. Luo and Y. Yang, J. Chem. Phys., 115, 2818-2826 (2001).

18) K. Luo and Y. Yang, Macromol. Theory Simul., 11, 429-437 (2002).

19) T. Xu, M. T. Shaw and R. A. Weiss, J. Polymer Sci. B, 42, 1725-1738 (2004).

20) K. Luo, H. Zhang and Y. Yang, Macromol. Theory Simul., 13, 335-344 (2004).

21) C. Lundell, E. H. A. de Hoog, R. H. Tromp, A.-M. Hermansson, J. Colloid Interface Sci., 288, 222-229 (2005).

22) P. A. Bhadane, M. F. Champagne, M. A. Huneault, F. Tofan and B. D. Favis, Polymer, 47, 2760-2771 (2006).

23) K. Luo, W. Gronski and C. Freidrich, Eur. Phys. J. E, 15, 177187 (2004)

24) S. A. Madbouly, T. Ougizawa and T. Inoue, Polymer, 45, 4483-4489 (2004).

25) M. K. Endoh, M. Takenaka and T. Hashimoto, Polymer, 47, 7271-7281 (2006) 